\title{
The non-anticoagulation costs of atrial fibrillation management: findings from an observational study in NHS Primary Care
}

\author{
George Kassianos, ${ }^{1}$ Chris Arden, ${ }^{2}$ Simon Hogan, ${ }^{3}$ Laura Baldock, ${ }^{4}$ Ahmet Fuat ${ }^{5}$ \\ The Ringmead Medical Practice, Bracknell, UK; ${ }^{2}$ Park Surgery, Chandlers Ford, UK; ${ }^{3}$ Sanofi, Guildford, UK; ${ }^{4} \mathrm{pH}$ Associates Ltd, Marlow, UK; \\ ${ }^{5}$ Carmel Medical Practice, Darlington, UK
}

\begin{abstract}
Background: Atrial fibrillation (AF) management represents a significant burden on the UK NHS. Understanding this burden will be important in informing future health care planning and policy development.

Aim: To describe the non-anticoagulation costs associated with AF management in routine UK clinical practice.

Materials, patients and methods: A retrospective observational study of 825 patients with AF undertaken in eight UK primary care practices. Data collected from routine clinical and prescribing records of all eligible, consenting patients, for a period of up to 3 years. The first 12 weeks following diagnosis was defined as the 'initiation phase'; the period after week 12 was defined as the 'maintenance phase'

Results: Mean (SD) total cost of AF management was $£ 941$ ( $£ 1094)$ per patient in the initiation phase and $£ 426$ ( $£ 597$ ) per patient-year in the maintenance phase. AF-related inpatient admissions contributed most to total costs; the mean (SD) total cost per patient in the initiation phase was $£ 2285$ ( $£ 900$ ) for admitted and $£ 278$ ( $£ 252$ ) for non-admitted patients. Mean maintenance phase costs per year were $£ 1323$ ( $£ 755)$ and $£ 168(£ 234)$, respectively, for admitted and non-admitted patients. Significant patient variables contributing to high cost in the initiation phase were hypertension and younger patient age, although only accounting for $6 \%$ of cost variability. Significant variables in the maintenance phase ( $18 \%$ of cost variability) were the presence of congestive heart failure, structural heart disease or diabetes and the frequency of day case admissions, ECGs and hospitalisations in the initiation phase.
\end{abstract}

Conclusions: Inpatient admissions contributed most to total AF management costs. Given the burden of hospital care, future work should focus on evaluating the appropriateness and reasons for hospitalisation in patients with AF and the factors affecting length of stay, with the aim of identifying opportunities to safely reduce inpatient costs.

A number of significant patient characteristics and initiation phase variables were identified, which accounted for $18 \%$ of the variability in total maintenance phase costs. However, none of these could adequately predict high maintenance phase costs.

Keywords: atrial fibrillation, resource use, primary health care, health care costs, observational study.

$\begin{array}{ll}\text { Citation } & \begin{array}{l}\text { Kassianos G, Arden C, Hogan S, Baldock L, Fuat A. The non-anticoagulation costs of atrial fibrillation management: findings from an } \\ \text { observational study in NHS Primary Care. Drugs in Context 2014; 3: 212254. doi: 10.7573/dic.212254 } \\ \text { Provenance }\end{array} \\ \text { Submitted; externally peer reviewed } \\ \text { Submitted: } 29 \text { April 2013; Accepted, subject to peer review: } 30 \text { April 2013; Revised manuscript submitted: } 18 \text { March 2014; } \\ \text { Published: } 9 \text { April } 2014 \\ \text { Copyright } & \begin{array}{l}\text { Commons Attribution License Deed CC BY NC ND 3.0 which allows anyone to copy, distribute and transmit the article provided it is } \\ \text { properly attributed in the manner specified by Drugs in Context. No other uses without permission. }\end{array} \\ \text { Correspondence address } & \text { Dr George Kassianos, The Ringmead Medical Practice, Birch Hill Medical Centre, Leppington, Bracknell, RG12 7WW, UK } \\ \text { Email address } & \text { gckassianos@btinternet.com } \\ \text { Abbreviations } & \text { A\&E, accident and emergency; AF, atrial fibrillation; ANOVA, Analysis of Variance; BNF, British National Formulary; CABG, coronary artery } \\ \text { bypass graft; CVA, cerebrovascular accident; CXR, chest x-ray; ECG, electrocardiogram; ECHO, echocardiogram; FBC, full blood count; } \\ \text { Gl, gastrointestinal; GP, general practitioner; GPwSI, general practitioner with special interest; MI, myocardial infarction; NHLI, National } \\ \text { Heart and Lung Institute; NHS, National Health Service; PR, per rectum; REC, research ethics committee; SD, standard deviation; } \\ \text { SOB, shortness of breath. }\end{array}$

\section{Introduction}

The management and treatment of atrial fibrillation (AF) represents a significant resource use burden on the UK National Health Service (NHS), both in primary and secondary care. The Office of Health Economics estimates that in 2008 the

Drugs in Context - The journal of interventions in clinical practice This full text PDF downloaded from www.drugsincontext.com Drugs in Context 2014; 3: 212254. ISSN 1740-4398 Copyright (C) 2014 Kassianos G, Arden C, Hogan S, Baldock L, Fuat A. Distributed under the terms of the Creative Commons Attribution License Deed CC BY NC ND 3.0. No other uses without permission. total direct cost of AF to the NHS was $£ 429$ million; there were approximately $851,095 \mathrm{GP}$ visits attributable to $\mathrm{AF}$ and patients with primary or secondary diagnoses of AF occupied an estimated 5.7 million bed-days [1]. The cost per AF-related stroke is estimated at $£ 11,900$ in the first year alone following stroke occurrence [2]. With the incidence and prevalence of AF predicted to rise significantly in the coming years due to an ageing population [3] and improving survival from underlying conditions closely associated with AF $[4,5,6]$, this economic burden has the potential to increase significantly unless management efficiencies are made. 
Given the economic burden of AF on the NHS, it is important that GPs and commissioners focus on measures to reduce AF-related health care costs while maintaining or improving quality of care, through service improvements and more effective diagnosis, treatment and management of patients with the condition, and effective anticoagulation. This will become increasingly important in the coming years, with the NHS under pressure to make significant efficiency savings and the UK government proposing major reforms which will change the way in which health care services are commissioned, organised and delivered.

A clear understanding of current AF management pathways and resource use will be important in informing future health care planning and policy development. Whilst existing data provide an estimate of the total cost of AF to the UK health care system, there are currently only limited international data [7-11] and no published UK studies describing the per-patient costs, differences between practices and the variables contributing to high resource use in 'real world' clinical practice.

The aim of this observational research study was to describe the non-anticoagulation NHS costs associated with the management of AF in routine UK clinical practice. No attempt has been made to describe the costs of anticoagulation for AF. There are many different models of anticoagulation care, including traditional secondary care models and primary care (GP)-based services $[12,13]$, and there is evidence of variation in the quality and effectiveness of these services throughout the country [12]. Anticoagulation therapy is a rapidly changing area of practice, with the advent of new oral anticoagulants and an expected increase in the uptake of anticoagulation. There have also been developments such as patient self-testing [14] and the National Institute for Health and Care Excellence (NICE) have recently issued commissioning guidance to support the redesign of local care pathways [12], taking account of NICE recommendations for the novel oral anticoagulants. With many changes expected in this area of practice in the forthcoming years, any description of anticoagulation costs based on current practice is likely to be quickly superseded.

\section{Materials and methods}

\section{Study design and methodology}

This observational study was conducted by retrospective review of the clinical records of patients diagnosed with AF in eight general medical practices in England $(n=7)$ and Wales $(n=1)$, selected to ensure a good geographical spread and a mixture of urban and rural practices. Brompton, Harefield and NHLI Research Ethics Committee (REC) and local management approval were given to conduct the study in the participating primary care trusts.

The general practitioner (GP) investigator at each site identified candidate patients by searching the electronic patient records for currently registered patients aged $\geq 18$ years at diagnosis, with a diagnosis of AF by Read codes for AF, AF with flutter, persistent AF, permanent AF and paroxysmal AF. Patient consent was sought via post by the GP for access to their records by a researcher, who then checked eligibility in the consenting patients against the remaining inclusion and exclusion criteria. Patients with secondary AF, those diagnosed $<12$ weeks before data collection or with no diagnosis date were excluded. A sample size of 1000 patients (500 initiation 500 maintenance) was chosen to ensure confidence intervals of $\pm 5 \%$ on estimates of proportions, also allowing for a cluster effect of 1.2 due to the multicentre design.

Anonymised coded data were collected retrospectively from the primary care clinical records to describe resource use including AF-related prescribing, primary and secondary care visits and hospitalisations and demographics. Data were collected between March and August 2010. For each patient, data were collected for the most recent 3 years of management (i.e. 2007-2010) or from the date of diagnosis of AF, if the patient was diagnosed $<3$ years before data collection.

For the purposes of this study the first 12 weeks of management following diagnosis was referred to as the initiation phase'. The period from week 12 onwards was referred to as the 'maintenance phase'. This distinction was made because it was recognised that AF resource use was likely to be higher in the period immediately following diagnosis. For patients who had been recently diagnosed with $\mathrm{AF}$ ( $<9$ months before data collection), data were collected on the initiation phase only. For patients diagnosed more than 3 years before data collection, data were collected on the most recent 3 years of management (i.e. maintenance phase only), whilst patients diagnosed 9 months-3 years before data collection provided data on both the initiation and maintenance phases of management.

\section{Analysis methodology}

Data were analysed using Microsoft Excel ${ }^{\circledR}$ and SPSS ${ }^{\circledR}$ according to a pre-agreed analysis plan. The main endpoint was NHS resource use, broken down by initiation and maintenance phases of management.

Resource use in the initiation phase is expressed as resource use per patient within a 12-week period. Resource use in the maintenance phase is expressed as resource use per patient-year, with data adjusted for patients providing between 6 and 12 months of maintenance data.

Costs were assigned to AF-related primary care visits, secondary care attendances, hospitalisations, investigations and blood tests, using published NHS reference costs [15-19] (Table 1). Where more than one reference cost was available for a particular aspect of management, the mean cost was used. A single cost (i.e. the national average unit cost for a non-elective inpatient admission for arrhythmia or conduction disorder) was assigned to each AF-related hospitalisation, irrespective of the length of stay or any procedures/investigations performed during the admission. The costs for AF medications were assigned using the prices published in BNF 61, March 2011 [20] (Table 2). Drug doses were not recorded for the study and therefore costs were assigned for each medication using a presumed daily dose (the midpoint of all the recommended doses published in BNF 61 for that medication). Route of administration was also not recorded for the study and therefore costs were assigned for oral 
Table 1. Costs assigned to each component of AF management [15-19].

\begin{tabular}{lc}
\hline Component of AF management & Cost assigned $(£)$ \\
\hline Primary and secondary care attendances & \\
Primary care visit & $£ 32.00$ \\
Outpatient visit & $£ 152.00$ \\
Day case admission* & $£ 637.00$ \\
Inpatient admission & $£ 1534.00$ \\
A\&E attendance & $£ 97.00$ \\
Investigations/procedures & \\
Electrocardiogram (ECG) & $£ 32.50$ \\
Holter ECG & $£ 59.79$ \\
Chest x-ray & $£ 86.52$ \\
Echocardiogram (ECHO) & $£ 76.00$ \\
Blood tests & \\
Electrolytes & $£ 4.11$ \\
Full blood count (FBC) & $£ 4.57$ \\
Renal function & $£ 4.11$ \\
Thyroid function test & $£ 3.68$ \\
\hline
\end{tabular}

*Patient admitted electively for care which does not require a hospital bed overnight.

Abbreviation

$\mathrm{AF}$, atrial fibrillation

doi: $10.7573 /$ dic.212254.t001

Table 2. Costs assigned to AF medications [20].

\begin{tabular}{lcc}
\hline Drug name & $\begin{array}{c}\text { Presumed } \\
\text { average daily dose }\end{array}$ & Average daily cost* \\
\hline Amiodarone & $200 \mathrm{mg}$ & $£ 0.16$ \\
Atenolol & $75 \mathrm{mg}$ & $£ 0.09$ \\
Bisoprolol & $10 \mathrm{mg}$ & $£ 0.25$ \\
Carvedilol & $25 \mathrm{mg}$ & $£ 0.23$ \\
Co-tenidone & $62.5 \mathrm{mg}$ & $£ 0.05$ \\
Digoxin & $125 \mathrm{mg}$ & $£ 0.03$ \\
Diltiazem & $240 \mathrm{mg}$ & $£ 0.38$ \\
Disopyramide & $500 \mathrm{mg}$ & $£ 1.15$ \\
Flecainide & $200 \mathrm{mg}$ & $£ 0.42$ \\
Labetalol & $400 \mathrm{mg}$ & $£ 0.26$ \\
Lercanidipine & $10 \mathrm{mg}$ & $£ 0.20$ \\
Metoprolol & $150 \mathrm{mg}$ & $£ 0.13$ \\
Nebivolol & $5 \mathrm{mg}$ & $£ 0.25$ \\
Nifedipine & $30 \mathrm{mg}$ & $£ 0.21$ \\
Propafenone & $450 \mathrm{mg}$ & $£ 0.24$ \\
Propranolol & $80 \mathrm{mg}$ & $£ 0.03$ \\
Sotalol & $240 \mathrm{mg}$ & $£ 0.22$ \\
Verapamil & $240 \mathrm{mg}$ & $£ 0.07$ \\
\hline
\end{tabular}

*Average of the minimum and maximum costs of all generic and proprietary versions.

Abbreviation

$\mathrm{AF}$, atrial fibrillation

doi: $10.7573 /$ dic.212254.t002 tablets/capsules, using the average of the minimum and maximum costs of all the generic and proprietary versions of each drug (Table 2).

The costs of AF-related primary and secondary care attendances, hospitalisations, investigations, blood tests and AF medications, were used to calculate a total cost per patient for the initiation and maintenance phases of management as applicable. All anticoagulation costs (i.e. visits for anticoagulation, INR tests and antiplatelet/anticoagulant medication) were excluded from the analysis.

Analysis of AF costs included stratification by type of AF, by centre, and by patients who did and did not require inpatient hospitalisation. Costs by AF type were analysed using Analysis of Variance (ANOVA).

Costing data were analysed using multiple regression to ascertain which combination of variables contributed most to the total costs during both the initiation and maintenance phases of management. Analysis of initiation phase costs was carried out for all patient-related independent variables; analysis of maintenance phase costs included both patient-related and initiation phase variables. Stepwise regression was carried out whereby at each stage the least significant variable of the previous stage was removed before the next variable that most contributed to the explanation of the variability of the dependent variable was added. This continued until no other variable contributed to the explanation with a significance of $p<0.05$.

\section{Results}

\section{Participating practices}

Eight general medical practices of 5-10 GPs with a median list size of 13,440 (range 6500-15,500) participated, with three practices including a GP with a special interest $(\mathrm{GPwSI})$ in the management of AF.

There were 1245 patients across all the participating practices who met the eligibility criteria and all were approached for consent. Data were collected from all 825 (66\%) consenting patients. The number of patients per practice ranged from 82 to 151. Data were available on the initiation phase from 310 patients and the maintenance phase from 769 patients. Two hundred and fifty-four patients provided data on both phases.

\section{Patient characteristics}

There were $462(56 \%)$ male patients. At the time of diagnosis $17 \%$ of patients (137/819 with data available) were aged less than 60 years and $682(83 \%)$ were aged 60 years or over. The mean age at diagnosis was 70.5 years (SD 11.1; range 22.4-95.7).

At the time of data collection $363(44.0 \%)$ patients had a current diagnosis of permanent $\mathrm{AF}$ (i.e. an ongoing long-term episode), 299 (36.2\%) paroxysmal AF (i.e. recurrent episodes that self-terminate in $<7$ days), 55 (6.7\%) persistent $\mathrm{AF}$ (i.e. recurrent episodes lasting $>7$ days) and $46(5.6 \%)$ patients first-detected $\mathrm{AF}$ (i.e. only one diagnosed episode). Fourteen (1.7\%) patients had a record of two types of AF and 48 (5.8\%) patients had no record of AF type. 
Table 3. Health care visits for AF.

\begin{tabular}{|c|c|c|c|c|}
\hline \multirow[b]{2}{*}{ Visit type } & \multicolumn{2}{|c|}{ Initiation phase $(n=310)$} & \multicolumn{2}{|c|}{ Maintenance phase $(n=769)$} \\
\hline & $\mathrm{N}(\%)$ of patients & $\begin{array}{l}\text { Mean no. visits per } \\
\text { patient (SD) }\end{array}$ & $\mathrm{N}(\%)$ of patients & $\begin{array}{c}\text { Mean no. visits per } \\
\text { patient-year (SD) }\end{array}$ \\
\hline \multicolumn{5}{|l|}{ AF-related primary care visits } \\
\hline Patients with any primary care visit & $253(82 \%)$ & $2.4(2.2)$ & $613(80 \%)$ & $1.5(1.8)$ \\
\hline - GP visits & $250(81 \%)$ & $2.3(2.1)$ & $581(76 \%)$ & $1.4(1.7)$ \\
\hline - Nurse visits & $45(15 \%)$ & $0.2(0.5)$ & $173(22 \%)$ & $0.2(0.3)$ \\
\hline \multicolumn{5}{|c|}{ AF-related secondary care visits (excluding inpatient admissions) } \\
\hline Patients with any secondary care visit (excl. admissions) & $196(63 \%)^{*}$ & $1.1(1.2)$ & $427(56 \%)$ & $0.8(1.0)$ \\
\hline - Outpatient clinic & $176(57 \%)$ & $0.8(0.9)$ & $384(50 \%)$ & $0.6(0.9)$ \\
\hline - Day case unit* & $12(4 \%)$ & $0.0(0.2)$ & $52(7 \%)$ & $0.0(0.2)$ \\
\hline - A\&E visit & $26(8 \%)$ & $0.1(0.5)$ & $82(11 \%)$ & $0.0(0.2)$ \\
\hline - Other secondary care visit & $3(<1 \%)$ & $0.0(0.1)$ & $2(<1 \%)$ & $0.0(0.0)$ \\
\hline \multicolumn{5}{|l|}{ AF-related inpatient admissions } \\
\hline Patients with any inpatient admission & $101(33 \%)$ & $0.4(0.7)$ & $139(18 \%)$ & $0.1(0.3)$ \\
\hline Mean (SD) length of hospital stay (days) & $5.6(7.8)$ & - & $6.4(9.6)$ & - \\
\hline
\end{tabular}

*A day case admission is where a patient is admitted electively for care which does not require a hospital bed overnight. The majority of day case admissions in this study were for electrical cardioversion.

\section{Abbreviations}

$A \& E$, accident and emergency; $A F$, atrial fibrillation; GP, general practitioner; $N / n$, number of patients; $S D$, standard deviation doi: $10.7573 /$ dic.212254.t003

Table 4. Number of patients hospitalised by reason. ${ }^{* 1}$

\begin{tabular}{|c|c|c|c|c|}
\hline \multirow[b]{2}{*}{ Recorded reason for hospitalisation } & \multicolumn{2}{|c|}{ Initiation phase $(n=101) * 2$} & \multicolumn{2}{|c|}{ Maintenance phase $(n=139) * 2$} \\
\hline & $\mathbf{N}$ & $\%$ & $\mathbf{N}$ & $\%$ \\
\hline $\mathrm{AF}$ & 25 & 25 & 11 & 8 \\
\hline Chest pain & 9 & 9 & 18 & 13 \\
\hline Pacemaker & 6 & 6 & 17 & 12 \\
\hline Palpitations & 9 & 9 & 10 & 7 \\
\hline Shortness of breath (SOB) & 8 & 8 & 10 & 7 \\
\hline Cerebrovascular accident (CVA) & 3 & 3 & 11 & 7 \\
\hline Cardiac ablation & 2 & 2 & 11 & 7 \\
\hline Fast AF & 5 & 5 & 7 & 5 \\
\hline Myocardial Infarction (MI) & 4 & 4 & 2 & 1 \\
\hline Angiogram & 3 & 3 & 2 & 1 \\
\hline Coronary artery bypass graft (CABG) & 2 & 2 & 3 & 2 \\
\hline Collapse & 1 & 1 & 4 & 3 \\
\hline Heart failure & 2 & 2 & 3 & 2 \\
\hline Chest infection & 4 & 4 & 0 & 0 \\
\hline Fall & 0 & 0 & 4 & 3 \\
\hline Palpitations + SOB & 3 & 3 & 1 & 1 \\
\hline Acute heart failure & 1 & 1 & 2 & 1 \\
\hline $\mathrm{AF}+$ congestive cardiac failure & 3 & 3 & 0 & 0 \\
\hline Angina & 2 & 2 & 1 & 1 \\
\hline Angiography & 1 & 1 & 2 & 1 \\
\hline Arrhythmia & 1 & 1 & 2 & 1 \\
\hline Breathlessness & 1 & 1 & 2 & 1 \\
\hline Chest pain + SOB & 1 & 1 & 2 & 1 \\
\hline Gl bleed & 0 & 0 & 3 & 2 \\
\hline PR bleed & 0 & 0 & 3 & 2 \\
\hline
\end{tabular}

*1Partial table showing reasons with 3 or more patients associated.

${ }^{* 2} \mathrm{n}$ is equal to the number of patients with one or more inpatient admissions in either the initiation or maintenance phase of management. Abbreviations

AF, atrial fibrillation; CABG, coronary artery bypass graft; CVA, cerebrovascular accident; Gl, gastrointestinal; MI, myocardial infarction; $\mathrm{N} / \mathrm{n}$, number of patients; $\mathrm{PR}$, per rectum; SOB, shortness of breath

doi: $10.7573 /$ dic.212254.t004 


\section{AF-related resource use}

Details of the numbers of visits to primary and secondary care, and inpatient hospital admissions are summarised in Table 3. Table 4 shows the number of inpatient hospitalisations by reason.
Table 5 shows the AF-related blood samples, investigations and procedures performed during both the initiation and maintenance phases of management. This includes the number and percentage of patients undergoing each test and the mean (SD) number performed per patient.

Table 5. AF-related blood tests and investigations. ${ }^{* 1}$

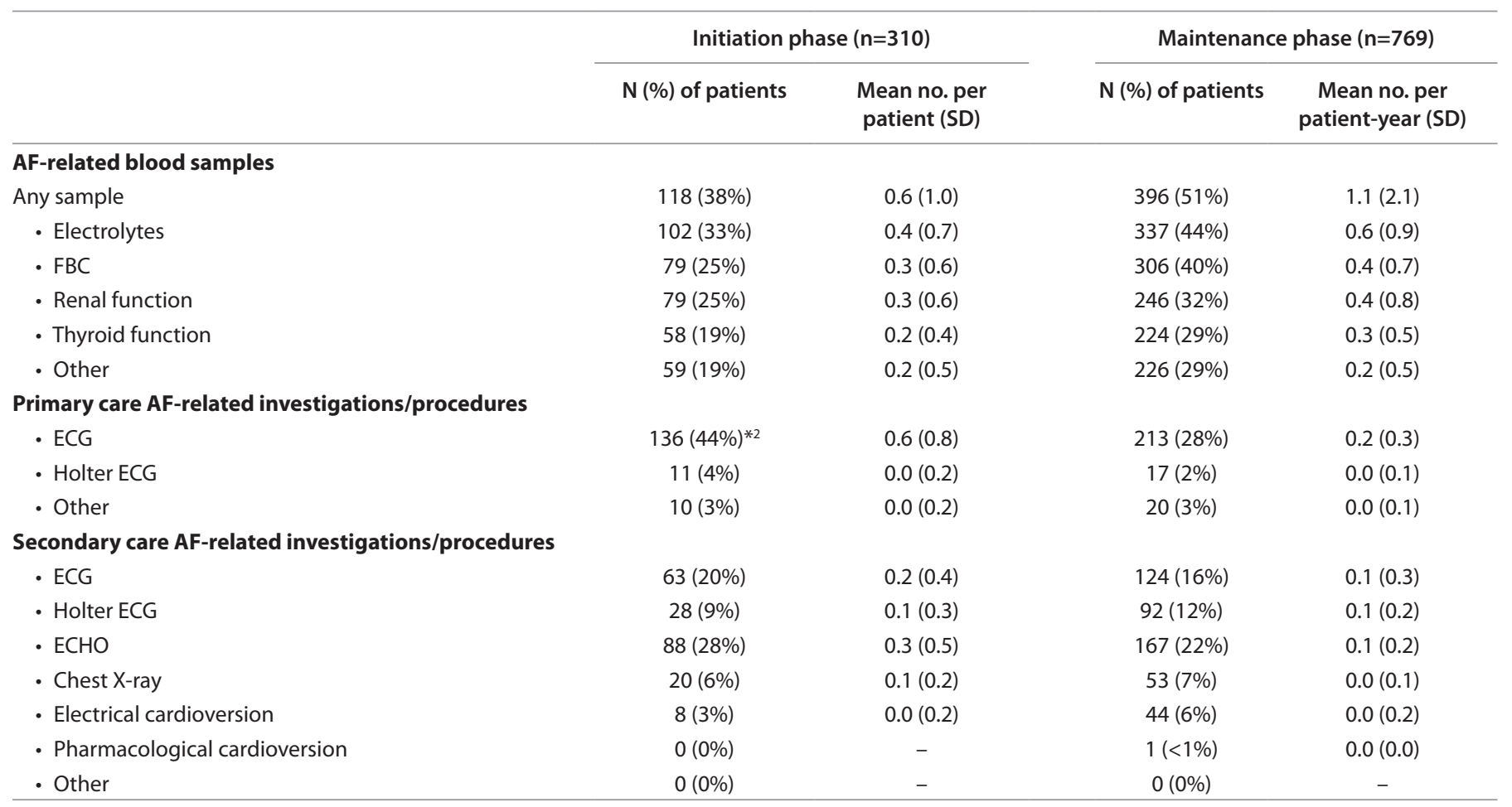

*1The number of blood tests and procedures recorded (in particular ECGs during the initiation phase) is likely to be underestimated, since some patients will have been diagnosed and had an ECG whilst in hospital (details of which may not have been recorded in the primary care records). In addition, a number of patients may have had ECGs carried out prior to diagnosis.

${ }^{* 2}$ All percentages are calculated out of the total number of patients (i.e. 310 in the initiation phase and 769 in the maintenance phase), NOT the number of patients who were referred to secondary care.

\section{Abbreviations}

AF, atrial fibrillation; ECG, electrocardiogram; ECHO, echocardiogram; $F B C$, full blood count; N/n, number of patients; SD, standard deviation doi: 10.7573/dic.212254.t005

Table 6. Cost of AF management.

\begin{tabular}{|c|c|c|c|c|c|c|}
\hline \multirow[b]{2}{*}{ Component of AF management } & \multicolumn{3}{|c|}{$\begin{array}{l}\text { Cost per patient during } \\
\text { initiation phase }(n=310)\end{array}$} & \multicolumn{3}{|c|}{$\begin{array}{l}\text { Cost per patient-year during } \\
\text { maintenance phase }(n=769)\end{array}$} \\
\hline & Mean & SD & Range* $^{*}$ & Mean & SD & Range \\
\hline Investigations (ECG, Holter ECG, ECHO, CXR) & $£ 59$ & $£ 60$ & $£ 0-£ 243$ & $£ 23$ & $£ 36$ & $£ 0-£ 240$ \\
\hline Blood testing & $£ 5$ & $£ 9$ & $£ 0-£ 50$ & $£ 7$ & $£ 11$ & $£ 0-£ 75$ \\
\hline AF medications & $£ 9$ & $£ 10$ & $£ 0-£ 47$ & $£ 49$ & $£ 49$ & $£ 0-£ 288$ \\
\hline AF-related primary care visits & $£ 78$ & $£ 69$ & $£ 0-£ 416$ & $£ 49$ & $£ 58$ & $£ 0-£ 365$ \\
\hline AF-related secondary care visits (outpatient, day case, A\&E) & $£ 166$ & $£ 201$ & $£ 0-£ 1426$ & $£ 128$ & $£ 206$ & $£ 0-£ 1526$ \\
\hline AF-related inpatient admissions & $£ 624$ & $£ 1006$ & $£ 0-£ 6136$ & $£ 170$ & $£ 448$ & $£ 0-£ 4768$ \\
\hline Total cost & £941 & $£ 1094$ & $£ 0-£ 7285$ & $£ 426$ & $£ 597$ & $£ 0-£ 4961$ \\
\hline
\end{tabular}

*Where the total cost for a patient in either the initiation or maintenance phase was zero, this may be due to patients being diagnosed and treatments initiated in secondary care (details of which may not have been recorded in the primary care records). In addition, a number of patients may have had investigations and primary or secondary care visits prior to diagnosis.

Abbreviations

$A \& E$, accident and emergency; AF, atrial fibrillation; CXR, chest x-ray; ECG, electrocardiogram; ECHO, echocardiogram; N/n, number of patients; SD, standard deviation doi: $10.7573 /$ dic.212254.t006 
Figure 1. Distribution of total cost per patient in initiation and maintenance phases of management.

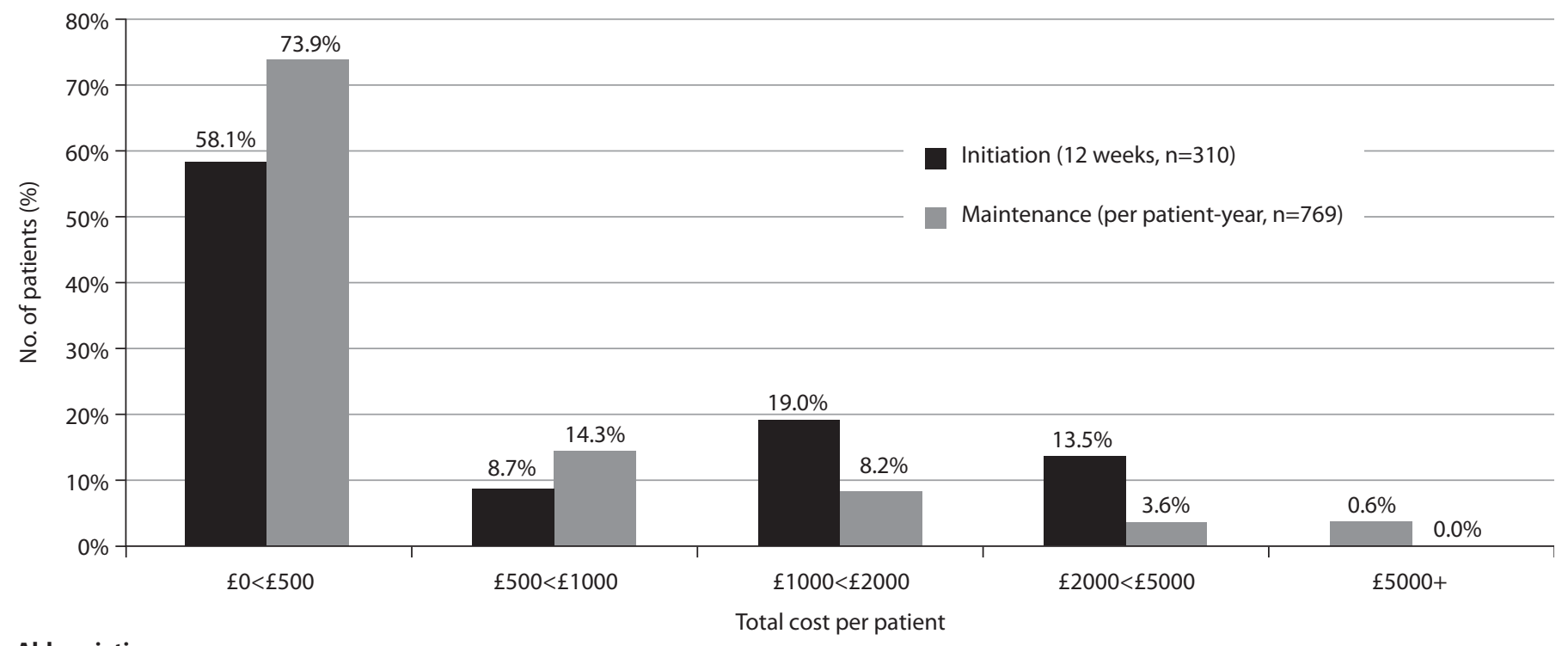

\section{Abbreviation}

$\mathrm{N} / \mathrm{n}$, number of patients

doi: 10.7573/dic.212254.f001

Table 7. Costs of AF management, stratified by patients with and without inpatient admissions.

\begin{tabular}{|c|c|c|c|c|c|c|c|c|}
\hline & \multicolumn{4}{|c|}{ Cost per patient during initiation phase } & \multicolumn{4}{|c|}{ Cost per patient-year during maintenance phase } \\
\hline Patients without inpatient admissions & 209 & $£ 278$ & $£ 252$ & $£ 0-£ 1716$ & 630 & $£ 168$ & $£ 234$ & $£ 0-£ 2016$ \\
\hline Patients with inpatient admission & 101 & $£ 2285$ & $£ 900$ & $£ 1534-£ 7256$ & 139 & $£ 1323$ & $£ 755$ & $£ 511-£ 4887$ \\
\hline
\end{tabular}

\section{Abbreviations}

$\mathrm{N} / \mathrm{n}$, number of patients; SD, standard deviation

doi: $10.7573 /$ dic.212254.t007

\section{Cost of AF management}

The estimated total cost of AF management per patient in both the initiation and maintenance phases, and the estimated cost for each separate aspect of AF management, is presented in Table 6 and Figure 1.

The mean total cost of AF management was $£ 941$ per patient in the initiation phase (SD $£ 1094$, range $£ 0-£ 7285$ ) and $£ 426$ per patient per year in the maintenance phase (SD $£ 597$, range $£ 0-£ 4961$ ). The cost of inpatient admissions contributed most to total management costs; the mean (SD) total cost of AF management per patient in the initiation phase was $£ 2285$ ( $£ 900$ ) for patients with inpatient hospitalisations during this period and $£ 278$ ( $£ 252$ ) for patients with no inpatient hospitalisations. The mean (SD) maintenance phase costs for admitted and nonadmitted patients, respectively, were $£ 1323$ (£755) and $£ 168$ (£234) (Table 7).

\section{Variables contributing to cost variability - multivariate analysis}

Table 8 shows the variables contributing most to total cost during both the initiation (Table 8 part a) and maintenance (Table 8 part b) phases of management. The regression coefficients show the added initiation phase cost $(\mathfrak{E})$ associated with each variable.

In the initiation phase, the variables contributing most to total cost were age at diagnosis (total initiation phase costs reduced by $£ 16$ with each increasing year of age) and hypertension (adding $£ 458$ to total costs) (Table 8 part a). However, these variables combined only explained $5.6 \%$ of the variability in initiation phase costs $\left(\mathrm{r}^{2}\right.$ coefficient of multiple determination=0.056) with the remaining $94.4 \%$ unknown (i.e. due to either random variation or factors which were not assessed in this study).

In the maintenance phase, the patient variables contributing most to total cost were the presence of congestive heart failure $(+£ 148)$, structural heart disease $(+£ 155)$ and diabetes $(+£ 180)$ (Table 8 part b). The presence of dyslipidaemia reduced maintenance phase costs by $£ 95$ per year. The numbers of hospitalisations, ECGs and day case admissions in the initiation phase were also associated with higher maintenance phase costs, with each hospitalisation adding $£ 360$, each ECG adding $£ 95$, and each day case admission adding $£ 807$ to the total cost for this period. All of these patient and initiation phase variables combined explained $18 \%$ of the variability in maintenance phase costs $\left(r^{2}\right.$ coefficient of multiple determination $\left.=0.183\right)$, with the 
Table 8. Variables contributing to total cost during the initiation and maintenance phases of management.

\begin{tabular}{|c|c|c|c|c|}
\hline & Regression coefficient $(£)^{*}$ & $95 \%$ Confidence interval $(£)( \pm)$ & Standard error & $p$ \\
\hline Age at diagnosis (per year) & -15.85 & 11.50 & 5.85 & 0.01 \\
\hline \multicolumn{5}{|c|}{ b) Patient characteristics and initiation phase variables contributing to total cost (per year) in maintenance phase } \\
\hline Presence of congestive heart failure & 148.20 & 104.87 & 53.42 & $<0.01$ \\
\hline Presence of dyslipidaemia & -94.54 & 87.63 & 44.64 & 0.034 \\
\hline $\begin{array}{l}\text { No. hospitalisations in initiation phase } \\
\text { (per hospitalisation) }\end{array}$ & 359.85 & 92.50 & 47.11 & $<0.0001$ \\
\hline No. of ECGs in initiation phase (per ECG) & 95.45 & 79.31 & 40.40 & 0.018 \\
\hline $\begin{array}{l}\text { No. day case admissions in initiation phase } \\
\text { (per day case admission) }\end{array}$ & 807.33 & 287.87 & 146.64 & $<0.0001$ \\
\hline
\end{tabular}

* Regression coefficients show the added initiation/maintenance phase cost associated with each patient or initiation phase variable.

Abbreviation

ECG, electrocardiogram

doi: 10.7573/dic.212254.t008

Table 9. Cost of AF management, stratified by AF type.

\begin{tabular}{|c|c|c|c|c|c|c|c|c|}
\hline AF type & \multicolumn{4}{|c|}{ Cost per patient during initiation phase } & \multicolumn{4}{|c|}{ Cost per patient-year during maintenance phase } \\
\hline First detected & 27 & $£ 981$ & $£ 887$ & $£ 0-£ 3317$ & 38 & $£ 108$ & $£ 348$ & $£ 0-£ 2025$ \\
\hline Persistent & 20 & $£ 585$ & $£ 688$ & $£ 0-£ 2237$ & 53 & $£ 467$ & $£ 660$ & $£ 0-£ 2598$ \\
\hline Permanent & 125 & $£ 959$ & $£ 1014$ & $£ 0-£ 3721$ & 348 & $£ 339$ & $£ 550$ & $£ 0-£ 4887$ \\
\hline
\end{tabular}

\section{Abbreviations}

$A F$, atrial fibrillation; ANOVA, analysis of variance; N/n, number of patients; SD, standard deviation doi: 10.7573/dic.212254.t009

remaining $82 \%$ unknown (i.e. due to either random variation or factors which were not assessed in this study).

\section{Cost of AF management by AF type}

The mean (SD) cost per patient in the initiation phase was $£ 981$ ( $£ 887$ ) for first detected AF, $£ 927$ (£1201) for paroxysmal, $£ 585$ ( $£ 688$ ) for persistent and $£ 959$ ( $£ 1014$ ) for permanent AF. Although the mean initiation phase cost for patients with persistent AF was lower than those of the other three groups, overall there was no significant difference in costs by AF type in the initiation phase $(p=0.53)$.

The mean (SD) cost per patient per year in the maintenance phase was $£ 108$ ( $£ 348$ ) for first detected, $£ 407$ ( 5584 ) for paroxysmal, $£ 467$ ( $£ 660$ ) for persistent and $£ 339$ ( $£ 550$ ) for permanent AF. Overall, there was a significant difference in maintenance phase costs per year by AF type $(p=0.005)$. The mean maintenance phase cost for patients with first detected AF was significantly lower than the cost for patients with all other AF types $(p<0.01)$, with no significant difference in cost between the subgroups of patients with paroxysmal, persistent and permanent AF (Table 9).

\section{Cost of AF management by centre}

The mean (SD) total cost of AF management per patient in the 12 -week initiation phase ranged between $£ 482$ (£611) (Centre 3) and $£ 1361$ ( $£ 1281$ ) (Centre 4). The mean (SD) cost per patient per year in the maintenance phase ranged from $£ 245$ (£279) (Centre 3) to $£ 630$ (£690) (Centre 5) (Figure 2). Table 10 shows the mean (SD) cost of each component of AF management, stratified by centre.

\section{Discussion}

This paper reports an analysis of the non-anticoagulation costs associated with the management of AF, from the results of a retrospective observational research study of 825 patients with AF, recruited from eight primary care practices in England and Wales. 
Figure 2. Mean (SD) cost per patient in initiation and maintenance phases of management - stratified by centre.

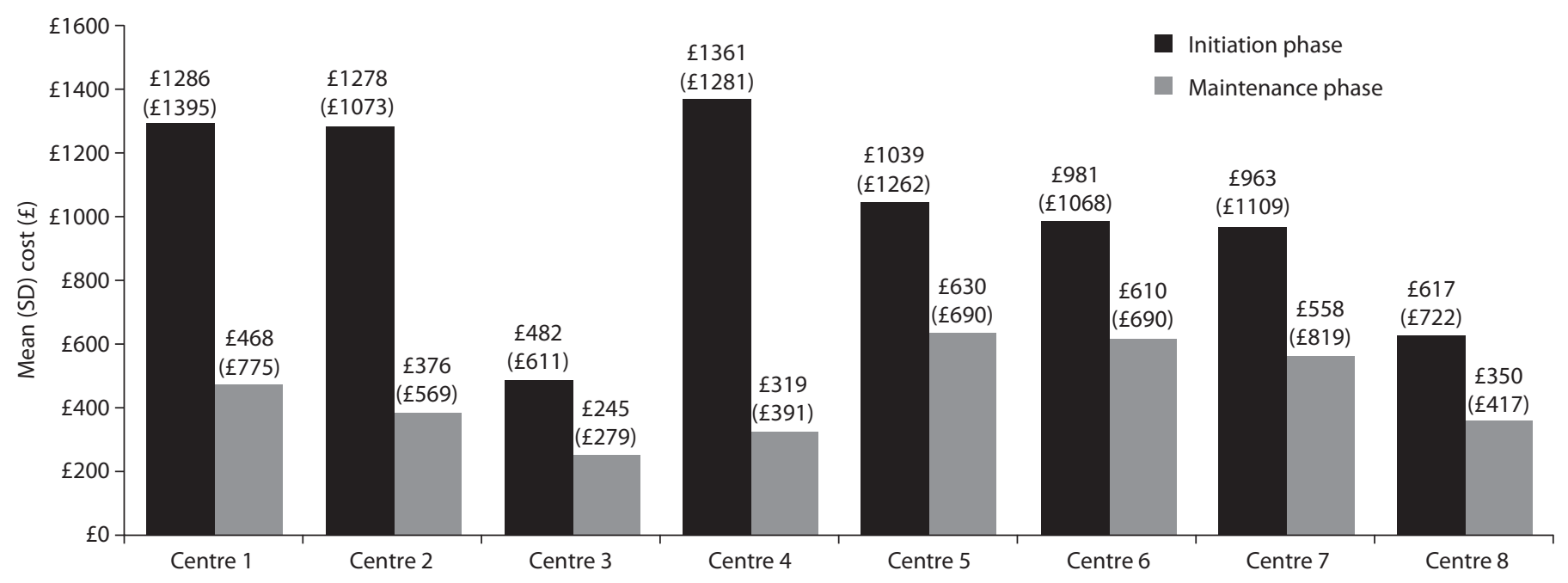

Abbreviation

$\mathrm{SD}$, standard deviation

doi: $10.7573 /$ dic.212254.f002

Table 10. Cost of each component of AF management, stratified by centre.

\begin{tabular}{|c|c|c|c|c|c|c|c|c|}
\hline $\begin{array}{l}\text { Component of } \\
\text { AF management }\end{array}$ & Centre 1 & Centre 2 & Centre 3 & Centre 4 & Centre 5 & Centre 6 & Centre 7 & Centre 8 \\
\hline \multicolumn{9}{|c|}{ Mean (SD) cost per patient during initiation phase } \\
\hline Blood tests & $£ 7(£ 9)$ & $£ 7(£ 9)$ & $£ 0(£ 0)$ & $£ 1(£ 3)$ & $£ 5(£ 7)$ & $£ 11(£ 13)$ & $£ 9(£ 10)$ & $£ 4(£ 7)$ \\
\hline AF medications & $£ 12(£ 10)$ & $£ 11(£ 11)$ & $£ 5(£ 8)$ & $£ 6(£ 6)$ & $£ 10(£ 10)$ & $£ 7(£ 9)$ & $£ 11(£ 11)$ & $£ 12(£ 11)$ \\
\hline Inpatient admissions & $£ 976(£ 1261)$ & $£ 920(£ 953)$ & $£ 195(£ 516)$ & $£ 1046(£ 1196)$ & $£ 736(£ 1169)$ & $£ 604(£ 1010)$ & $£ 575(£ 1031)$ & $£ 393(£ 679)$ \\
\hline \multicolumn{9}{|c|}{ Mean (SD) cost per patient-year during maintenance phase } \\
\hline Investigations & $£ 22(£ 37)$ & $£ 22(£ 31)$ & $£ 9(£ 17)$ & $£ 21(£ 28)$ & $£ 33(£ 49)$ & $£ 44(£ 50)$ & $£ 20(£ 36)$ & $£ 22(£ 32)$ \\
\hline Blood tests & $£ 6(£ 7)$ & $£ 5(£ 6)$ & $£ 0.09(£ 0.75)$ & $£ 0.24(£ 1.16)$ & $£ 12(£ 9)$ & $£ 27(£ 17)$ & $£ 6(£ 9)$ & $£ 3(£ 5)$ \\
\hline AF medications & $£ 71(£ 62)$ & $£ 51(£ 47)$ & $£ 40(£ 41)$ & $£ 34(£ 34)$ & $£ 51(£ 56)$ & $£ 43(£ 50)$ & $£ 54(£ 42)$ & $£ 51(£ 53)$ \\
\hline
\end{tabular}

\section{Abbreviations}

AF, atrial fibrillation; SD, standard deviation

doi: 10.7573/dic.212254.t010

As expected, inpatient admissions and secondary care attendances were the highest-costing components of AF management; the mean total cost per patient in the initiation phase was $£ 2285$ for patients with one or more inpatient hospitalisations during this period and just $£ 278$ for those with no inpatient hospitalisations. Findings for the maintenance phase were similar, with a mean cost per patient-year of $£ 1323$ and $£ 168$, respectively, for hospitalised and non-hospitalised patients. This finding is in line with results of previous studies conducted in the USA [10] and other European countries [7-9,11], which also found hospitalisations to contribute most to overall costs. With one-third of patients in the current study (33\%) admitted to hospital during the initiation phase and $18 \%$ during the maintenance phase, mean lengths of stay of 5.6 and 6.4 days (initiation and maintenance phase, respectively), and recently published work suggesting that AF-related hospitalisations are increasing at a faster rate than those for other cardiovascular diseases [21], hospital care for patients with AF clearly represents a considerable burden of health care resource and is an area which may be targeted to reduce overall costs. However, to ensure that cost-cutting measures do not compromise quality of care, further research is needed to evaluate the appropriateness and reasons for hospital admissions in patients with AF and the factors affecting length of stay; often it may be the patient's other comorbidities or social care needs which are the determining and limiting factor. 
The between-patient range of costs was high, with the care of most patients $58 \%$ in the initiation phase and $74 \%$ in the maintenance phase) costing little ( $<£ 500$ per patient/per patientyear) and a only a small number of patients ( $14 \%$ in the initiation phase and $4 \%$ in the maintenance phase) at the high end of the distribution (i.e. care costing more than $£ 2000$ per patient/ per patient-year). Whilst inpatient admissions were the highestcosting individual component of $\mathrm{AF}$ management, most patients had no inpatient admissions in either phase of management.

The presence of a small number of patients at the high end of the cost distribution led to our attempt (using multiple regression analysis) to identify factors predictive of high cost. The patient and initiation phase variables which correlated most with maintenance phase costs (accounting for 18\% of variability) were the presence of congestive heart failure, structural heart disease and diabetes, and the number of hospitalisations, ECGs and day case admissions in the initiation phase. The correlation between cardiac comorbidities and higher maintenance phase costs is consistent with the findings of studies conducted previously in other European countries $[7,9]$. However, it is very difficult to separate AF-related resource from that which is attributable to other comorbidities, and although the aim of this study was to record only AF-related resource, hospitalisations associated with other cardiac comorbidities (where AF was a secondary diagnosis or contributing factor) were sometimes also recorded. In these cases it is not known how much the diagnosis of AF contributed either to the initial hospital admission or length of stay. The correlation between initiation phase resource use (number of hospitalisations and day case admissions) is interesting to note, and suggests that patients who require secondary care intervention (particularly planned day case admissions) in the first 12 weeks following diagnosis will continue to have high costs in the period following this and may therefore warrant more intensive primary care management to try to avoid unplanned secondary care resource use. It should be noted, however, that only a small proportion of patients (4\%) had day case admissions in the initiation phase. The patient variables contributing most to total costs in the initiation phase were younger patient age and the presence of hypertension. However, these variables combined explained only a very small proportion $(6 \%)$ of the cost variability for this period. The correlation between younger patient age and increased initiation phase costs may indicate relatively more intensive management in younger patient groups, perhaps in terms of more frequent primary care review, earlier referral into secondary care or more intensive medication management. The finding cannot be explained by the higher use of interventions such as electrical cardioversion and cardiac ablation, as the use of these procedures was very low overall but particularly so in the initiation phase.

Analysis of AF management costs by AF type showed that although the mean initiation phase cost was lower for persistent $\mathrm{AF}$ (mean £585) than for paroxysmal (mean £927), permanent (mean £959) or first detected AF (mean £981), there was overall no significant difference between the groups $(p=0.531)$. In the maintenance phase the mean cost per patient per year was significantly lower for patients with first detected AF (£108) than for patients in any other AF category $(p<0.01)$. However, this is not surprising given that patients in this category have had (by definition) only one diagnosed AF episode. There were no significant differences in maintenance phase costs between the subgroups of patients with paroxysmal, persistent and permanent AF, suggesting that once an appropriate treatment strategy is determined and patients are established on medication, the management costs for the different types of AF are comparable.

There was wide variation in initiation and maintenance phase costs between centres, with mean initiation phase costs per patient ranging from $£ 482$ (Centre 3) to $£ 1361$ (Centre 4) and mean maintenance phase costs per patient per year ranging from $£ 245$ (Centre 3) to $£ 630$ (Centre 5).

\section{Strengths and limitations}

The primary strength of this study is that it provides a description of the costs of AF management in 'real world' UK clinical practice; however, there are a number of limitations. As data were collected retrospectively from primary care records, the data quality relied heavily on the level of detail routinely recorded in these records and on the information provided in correspondence from secondary care.

The number of blood tests and procedures recorded (in particular ECGs during the initiation phase) is likely to be underestimated, since some patients will have been diagnosed and had an ECG whilst in hospital (details of which may not have been recorded in the primary care records). In addition, a number of patients may have had ECGs carried out prior to diagnosis.

This study may provide an overestimate of the cost of hospital care in patients with AF. Data collectors were instructed to record 'AF-related' inpatient admissions; however, it is very difficult to separate AF-related resource from that which is attributable to other comorbidities, and for some recorded hospitalisations AF may have been a secondary diagnosis.

There are also limitations with the costing data. Drug costs were assigned using the BNF reference costs and it is acknowledged that these may not reflect the NHS purchase price. Data on drug doses and route of administration were not captured during the study and therefore the cost assigned to each medication was based on the cost of oral tablets/capsules for an assumed average daily dose. The average of the minimum and maximum costs of all generic and proprietary versions of each drug was used in calculations.

Inpatient stays were costed per episode, using a single cost assigned to an AF-related inpatient stay, and therefore the varying lengths of hospital stay and any operations, procedures or investigations performed during the admission, were not accounted for.

\section{Conclusions}

This study confirms that inpatient admissions and secondary care attendances are the largest contributors to total $\mathrm{AF}$ management costs, both in the first 12 weeks after diagnosis and in the period following this. Whilst this finding is not unexpected, it is helpful to quantify, and will be useful to commissioners and health care providers in discussions about how AF management can be made as efficient as possible. Given the burden of hospital care, health care professionals should review the appropriateness and reasons 
for hospital admissions in patients with AF and the factors affecting length of stay. Future work should focus on identifying opportunities to safely reduce avoidable hospital admissions and length of hospital stay, ensuring that patients are still referred to secondary care when appropriate. Further research is also warranted to explore the link between cost and patient outcomes, as this was beyond the scope of the current study.

The study identified a number of significant patient characteristics and initiation phase variables, which accounted for $18 \%$ of the variability in total maintenance phase costs. However, they could not predict high resource users in the maintenance phase and hence identify those for whom greater primary care intervention may reduce hospitalisations.

\section{Contributions}

The following were involved in the planning, conduct of the research study, interpretation and reporting of the results and met the criteria of authorship: Dr George Kassianos, The Ringmead Medical Practice, Bracknell; Dr Chris Arden, Park Surgery, Chandlers Ford; and Dr Ahmet Fuat, Carmel Medical Practice, Darlington.

Sanofi UK, guarantor, provided planning, took responsibility as the Sponsor and funder of the research and were involved in the planning, design and interpretation of the results and facilitated the conduct of the study.

$\mathrm{pH}$ Associates were commissioned by the Study Sponsor Sanofi UK to provide support with the design, conduct, analysis and reporting of study results and as scientific editorial support.

\section{Potential conflicts of interest}

International Committee of Medical Journal Editors' (ICMJE) Potential Conflicts of Interests forms for each of the authors are summarised below. Original forms for each of the authors are available for download at: http://drugsincontext.com/download/ dic.212254_COI.pdf

GK: reports receiving consulting fees from Sanofi UK.

CA: reports receiving consulting fees from Sanofi UK.

$\mathrm{SH}$ : is a full-time employee of Sanofi UK.

LB: is a full-time employee of $\mathrm{pH}$ Associates Ltd under commission by Sanofi UK.

AF: reports no competing interests.

\section{Funding declaration}

The research was funded by Sanofi UK, One Onslow Street, Guildford GU1 4YS, UK.

\section{Acknowledgments}

The authors wish to thank the following participating research centres who provided data, but do not fulfil the criteria for authorship: Dr John Ryan - The Alverton Practice, Penzance; Dr John Jones - Cyncoed Medical Centre, Cardiff; Dr Warwick Coulson Albany House Medical Centre, Wellingborough; Dr Trevor
Gooding - The Atherstone Surgery, Atherstone; and Dr Barbara King - Carterknowle \& Dore Medical Practice, Sheffield.

This study has been presented previously as a poster at the International Society for Pharmacoeconomics and Outcomes Research 15th Annual European Congress, Berlin, Germany, 3-7 November 2012.

\section{References}

1. The Office of Health Economics. Estimating the direct costs of atrial fibrillation to the NHS in the constituent countries of the UK and at SHA level in England, 2008. London: The Office of Health Economics; 2009.

2. Kerr M. Atrial Fibrillation Cost Benefit Analysis. NHS Improvement 2008:1-17.

3. Go A, Hylek E, Phillips K, et al. Prevalence of diagnosed atrial fibrillation in adults: national implications for rhythm management and stroke prevention: the AnTicoagulation and Risk Factors in Atrial Fibrillation (ATRIA) Study. JAMA 2001;285:2370-5.

http://dx.doi.org/10.1001/jama.285.18.2370

4. MacIntyre K, Capewell S, Stewart S, et al. Evidence of improving prognosis in heart failure: trends in case fatality in 66,547 patients hospitalized between 1986 and 1995 . Circulation 2000;102:1126-31. http://dx.doi.org/10.1161/01.CIR.102.10.1126

5. Capewell S, MacIntyre K, Stewart S, et al. Age, sex and social trends in out-of-hospital cardiac deaths in Scotland 1986-1995: a retrospective cohort study. Lancet 2001;358(9289):1213-7. http://dx.doi.org/10.1016/S0140-6736(01)06343-7

6. Rosamond W, Chambless L, Folsom A, et al. Trends in the incidence of myocardial infarction and in mortality due to coronary heart disease, 1987 to 1994 . N Engl J Med 1998;339:861-7. http://dx.doi.org/10.1056/NEJM199809243391301

7. Le Heuzey J, Paziaud O, Piot O, et al. Cost of care distribution in atrial fibrillation patients: the COCAF study. Am Heart J 2004;147:121-6. http://dx.doi.org/10.1016/S0002-8703(03)00524-6

8. Ringborg A, Nieuwlaat R, Lindgren P, et al. Costs of atrial fibrillation in five European countries: results from the Euro Heart Survey on atrial fibrillation. Europace 2008;10:403-11.

http://dx.doi.org/10.1093/europace/eun048

9. Holstenson E, Ringborg A, Lindgren P, et al. Predictors of costs related to cardiovascular disease among patients with atrial fibrillation in five European countries. Europace 2011;13:23-30. http://dx.doi.org/10.1093/europace/euq325

10. Reynolds MR, Essebag V, Zimetbaum P, Cohen DJ. Healthcare resource utilization and costs associated with recurrent episodes of atrial fibrillation: The FRACTAL Registry. J Cardiovasc Electrophysiol 2007;18:628-633. http://dx.doi.org/10.1111/j.1540-8167.2007.00819.x

11. McBride D, Mattenklotz AM, Willich SN, Bruggenjurgen $B$. The costs of care in atrial fibrillation and the effect 
of treatment modalities in Germany. Value Health 2009; 12:293-301.

http://dx.doi.org/10.1111/j.1524-4733.2008.00416.x

12. National Institute for Health and Care Excellence (NICE). Support for commissioning: anticoagulation therapy. May 2013. Available at: http://publications.nice.org.uk/ support-for-commissioning-anticoagulation-therapy-cmg49 [Last accessed 20 September 2013].

13. Rose P, James R, Chapman O, Marshall S. A real world evaluation to describe the characteristics, outcomes and resource use associated with patients being managed by a secondary care based anticoagulation service. ISPOR 14th Annual European Congress, 5-8 Nov 2011, Madrid, Spain [Abstract PCV128].

14. Fitzmaurice DA, Murray ET, McCahon D, et al. Self management of oral anticoagulation: randomised trial. BMJ 2005;331(7524):1057. http://dx.doi.org/10.1136/bmj.38618.580903.AE

15. Department of Health. National Schedule of Reference Costs 2009-10 for NHS Trusts. 2011. Available at: https://www.gov.uk/government/publications/nhsreference-costs-2009-2010 [Last accessed 24 March 2014 ].
16. NHS Kidney Care. Developing Robust Reference Costs for Kidney Transplantation in Adults, Final Report. 2010:1-40.

17. National Institute for Health and Clinical Excellence (NICE). Chest pain of recent onset: assessment and diagnosis of recent onset chest pain or discomfort of suspected cardiac origin, CG95. 2010. Available at: http://www.nice. org.uk/CG95 [Last accessed 21 March 2014].

18. Beckett G, Toft A. First-line thyroid function tests - TSH alone is not enough. Clin Endocrinol (Oxf) 2003;58:20-1. http://dx.doi.org/10.1046/j.1365-2265.2003.01690.x

19. Personal Social Services Research Unit (PSSRU). Unit costs of Health and Social Care 2010. Available at: http:// www.pssru.ac.uk/uc/uc2010contents.htm [Last accessed 19 March 2013].

20. Joint Formulary Committee. British National Formulary. Edition 61. London: BMJ Group and Pharmaceutical Press; 2011.

21. Keech M, Punekar Y, Choy A-M. Trends in atrial fibrillation hospitalisation in Scotland: an increasing cost burden. Br J Cardiol 2012;19:173-7.

http://dx.doi.org/10.5837/bjc.2012.031 